\title{
Quality of life in diabetic subjects with respect to metabolic syndrome: a case control study
}

\author{
Mahesh V.*, Latha K., Muninarayana C., Ravishankar S.
}

Department of Community Medicine, Sri Devaraj Urs Medical College, Kolar, Karnataka, India

Received: 02 August 2016

Accepted: 06 August 2016

\section{*Correspondence:}

Dr. Mahesh V.,

E-mail: maheshpsm1984@gmail.com

Copyright: ( ) the author(s), publisher and licensee Medip Academy. This is an open-access article distributed under the terms of the Creative Commons Attribution Non-Commercial License, which permits unrestricted non-commercial use, distribution, and reproduction in any medium, provided the original work is properly cited.

\section{ABSTRACT}

Background: Diabetes is a chronic non-communicable disease, leading to many complications and disability. With co morbidities it leads to a substantial decrease in the patients' quality of life (QoL). In diabetics, psychosocial factors such as depression are stronger predictors of medical outcomes such as hospitalization and death than are physical and metabolic factors such as presence of complications, body mass index, or HbA1c level. Hence this study was conducted with the objectives to correlate the quality of life $(\mathrm{QoL})$ domains with metabolic syndrome parameters among diabetic patients.

Methods: Hospital based case control study was done for a period of 3 months among 180 diabetics and nondiabetics in a rural tertiary care centre. Information on socio- demographic profile, diabetic history was collected using a pretested and structured questionnaire. Quality of life was assessed by WHOQOL-BREF. Statistical analysis was carried out by using SPSS 22 Version. Chi-square and independent t-test was the statistical test.

Results: Majority of subjects in both groups were females, $56.7 \%$ and $54.4 \%$ in cases and controls respectively. Mean age of cases and control was $57.9 \pm 11.7$ years and $57.4 \pm 11.2$ years respectively. Significant association between diabetes and metabolic syndrome was observed. Quality of life was significantly low among diabetics with metabolic syndrome than without metabolic syndrome.

Conclusions: Quality of life with respect to physical, psychological and social domain among diabetics with metabolic syndrome was reduced significantly.

Keywords: Quality of life, Diabetes mellitus, Metabolic syndrome

\section{INTRODUCTION}

WHO had defined Quality of life (QoL) as individuals' perceptions of their position in life in the context of the culture and value systems in which they live and in relation to their goals, expectations, standards and concerns. ${ }^{1}$ QoL is affected in chronic diseases due to various factors as reviewed by literature. ${ }^{2}$ Diabetes is a chronic non-communicable disease, leading to many complications and disability. With co morbidities it leads to a substantial decrease in the patients' quality of life (QoL). Health-related quality of life and diabetes-specific quality of life represent increasingly narrower concepts, there is currently no "gold standard" for the assessment diabetes-specific quality of life. In diabetics, psychosocial factors such as depression are stronger predictors of medical outcomes such as hospitalization and death than are physical and metabolic factors such as presence of complications, body mass index, or HbA1c level. ${ }^{3}$ Hence this study was conducted with the objectives to find the difference in quality of life (QoL) domains with metabolic syndrome among diabetic and non-diabetic subjects. 


\section{METHODS}

A hospital based case control study was conducted in a rural tertiary care centre of South India for a period of 6 months. Informed consent was obtained from type 2 Diabetic patients and controls were age and gender matched. Sample size of 180 was obtained at $80 \%$ power and $95 \%$ CI (two sided) by using the environmental domain $(71.2 \pm 12.1$ in cases $74.7 \pm 11.3$ in controls) QoL between cases and controls from the pilot study which gave the maximum sample size. A pretested and structured questionnaire was used to obtain the information on socio- demographic profile, diabetic history. The quality of life was assessed by WHOQOLBREF scale. ${ }^{4,5}$ Four domains of quality of life measured by using the above scale are: physical, psychological, social and environment, through a set of 26 items that can be self-administered. 5-point Likert scale was used to record the responses. Metabolic parameters such as fasting blood sugar, waist circumference, triglycerides, HDL, blood pressure were measured by standard techniques. Metabolic syndrome was diagnosed by increased waist circumference (with respect to male and female for South Asian criteria) and presence of two raised parameters such as FBS $(>100 \mathrm{mg} / \mathrm{dl}), \mathrm{SBP} \geq 130$ mmhg, DBP $\geq 85 \mathrm{mmhg}$, HDL ( $<40$ in males and $<50$ in females) and triglycerides $(\geq 150 \mathrm{mg} / \mathrm{dl}) .{ }^{6-8}$ Statistical Analysis was done using SPSS 22 software. Chi-square was the test of significance Qualitative data and Independent t-test was test of significance for difference in means between two groups.

\section{RESULTS}

In the study 180 cases (with diabetes mellitus) and controls (without diabetes mellitus) were included in the study to observe the difference in WHO BREF QoL with respect to metabolic syndrome.

Table 1: Socio demographic, metabolic syndrome and WHO BREF QOL profile of cases and controls in the study.

\begin{tabular}{|c|c|c|c|c|c|c|c|c|}
\hline & & & \multicolumn{5}{|c|}{ Group } & \multirow{3}{*}{$P$ value } \\
\hline & & & \multirow[t]{2}{*}{ Count } & Cases & \multicolumn{3}{|c|}{ Controls } & \\
\hline & & & & Mean & SD & Mean & SD & \\
\hline \multicolumn{3}{|l|}{ Age } & 180 & 57.9 & 11.7 & 57.4 & 11.2 & 0.655 \\
\hline \multirow{6}{*}{$\begin{array}{l}\text { Metabolic } \\
\text { syndrome } \\
\text { parameters }\end{array}$} & \multicolumn{2}{|c|}{ SBP } & 180 & 148.3 & 21.0 & 143.2 & 20.6 & $0.022 *$ \\
\hline & \multicolumn{2}{|c|}{ DBP } & 180 & 84.8 & 11.9 & 82.8 & 12.0 & 0.114 \\
\hline & \multicolumn{2}{|c|}{ WC } & 180 & 89.2 & 10.7 & 86.4 & 12.6 & $0.022 *$ \\
\hline & \multicolumn{2}{|c|}{ TG } & 180 & 255.7 & 115.4 & 209.5 & 101.7 & $<0.001 *$ \\
\hline & \multicolumn{2}{|c|}{ HDL } & 180 & 39.2 & 13.8 & 41.2 & 11.7 & 0.138 \\
\hline & \multicolumn{2}{|c|}{ FBS } & 180 & 160.4 & 42.98 & 106.3 & 22.1 & $<0.001 *$ \\
\hline \multirow{4}{*}{$\begin{array}{l}\text { WHO BREF } \\
\text { QoL domains }\end{array}$} & \multicolumn{2}{|c|}{ Physical } & 180 & 53.3 & 15.5 & 63.3 & 13.3 & $<0.001$ \\
\hline & \multicolumn{2}{|c|}{ Psychological } & 180 & 53.5 & 13.6 & 63.6 & 14.4 & $<0.001 *$ \\
\hline & \multicolumn{2}{|c|}{ Social } & 180 & 50.8 & 14.8 & 57.7 & 21.2 & $<0.001 *$ \\
\hline & \multicolumn{2}{|c|}{ Environment } & 180 & 70.9 & 13.4 & 72.8 & 11.3 & 0.141 \\
\hline \multirow{2}{*}{\multicolumn{2}{|c|}{ Metabolic syndrome }} & Absent & 155 & 67 & $37.2 \%$ & 88 & $48.9 \%$ & \multirow{2}{*}{$0.025 *$} \\
\hline & & Present & 205 & 113 & $62.8 \%$ & 92 & $51.1 \%$ & \\
\hline
\end{tabular}

Table 2: Comparison of WHO BREF QoL domains with metabolic syndrome among cases and controls.

\begin{tabular}{|c|c|c|c|c|c|c|c|c|c|c|}
\hline & \multicolumn{10}{|c|}{ Metabolic syndrome } \\
\hline & \multicolumn{5}{|c|}{ Present } & \multicolumn{5}{|l|}{ Absent } \\
\hline & \multicolumn{5}{|c|}{ Group } & \multicolumn{5}{|l|}{ Group } \\
\hline & \multicolumn{2}{|l|}{ Cases } & \multicolumn{2}{|c|}{ Controls } & \multirow{2}{*}{$P$ value } & \multicolumn{2}{|l|}{ Cases } & \multicolumn{2}{|c|}{ Controls } & \multirow{2}{*}{$P$ value } \\
\hline & Mean & SD & Mean & SD & & Mean & SD & Mean & SD & \\
\hline Physical & 47.3 & 14.9 & 63.3 & 13.0 & $<0.001 *$ & 63.5 & 10.3 & 63.3 & 13.6 & 0.920 \\
\hline Psychological & 48.6 & 13.2 & 65.0 & 14.1 & $<0.001 *$ & 61.7 & 9.8 & 62.1 & 14.6 & 0.831 \\
\hline Social & 46.1 & 14.3 & 58.6 & 21.8 & $<0.001 *$ & 58.8 & 12.1 & 56.8 & 20.6 & 0.477 \\
\hline Environment & 71.5 & 13.1 & 72.6 & 12.8 & 0.527 & 69.9 & 13.9 & 73.0 & 9.5 & 0.102 \\
\hline
\end{tabular}

Mean QoL of various domains such as physical (47.3 \pm 14.9$)$, psychological $(48.6 \pm 13.2)$, social domain $(46.1 \pm 14.3)$ among cases with metabolic syndrome and in controls physical domain (63.3 \pm 13$)$, psychological $(65 \pm 14.1)$, social domain $(58.6 \pm 21.8)$. This difference between cases and controls was statistically significant. 
With respect to environmental domain there was no significant difference in mean values between cases and controls in subjects with metabolic syndrome was significant. No significant difference in QoL parameters was observed between cases and controls in subjects with out metabolic syndrome (Table 2). Majority of subjects in both groups were females, $56.7 \%$ and $54.4 \%$ in cases and controls respectively. Mean age of cases was $57.9 \pm 11.7$ years and controls were $57.4 \pm 11.2$ years. There was no significant difference in mean age and gender between two groups. Significant difference was observed in mean SBP, waist circumference, triglycerides and FBS. No significant difference was observed in DBP and HDL between two groups. In the study 113 subjects $(62.8 \%)$ of cases and 92 subjects $(51.1 \%)$ of controls had metabolic syndrome. There was significant association between diabetes and metabolic syndrome (Table 1).

\section{DISCUSSION}

Diabetes being a chronic disease can influence overall quality of life and also can influence coping with their disease in short and long term successfully. In this regard a case control study was conducted to find the quality of life among diabetics and non-diabetics. Metabolic syndrome was looked as parameter which can influence quality of life among them. Overall 180 cases and 180 controls were included, all the subjects were subjected to metabolic syndrome evaluation.

Distribution of subjects in both groups majority of subjects were females, $56.7 \%$ and $54.4 \%$ in cases and controls respectively. Mean age of cases was $57.9 \pm 11.7$ years and controls were $57.4 \pm 11.2$ years. There was no significant difference in mean age and gender between two groups. Hence age and gender matching was attained to avoid confounding bias in the study. Socio demographic profile of subjects were similar to the studies conducted by Harish et al, Rubin et al. ${ }^{9-11}$

Parameters included in the metabolic syndrome were higher in Cases compared to controls as expected by the disease status. Whereas no difference was observed between cases and controls with diastolic blood pressure (DBP) and HDL. Significant association between diabetes and metabolic syndrome was observed.

Mean QoL of various domains such as physical (47.3 \pm 14.9$)$, psychological $(48.6 \pm 13.2)$, social domain (46.1 \pm 14.3$)$ among cases with metabolic syndrome and in controls Physical domain $(63.3 \pm 13)$, psychological $(65 \pm 14.1)$, social domain $(58.6 \pm 21.8)$. This difference between cases and controls was statistically significant. With respect to environmental domain there was no significant difference in mean values between cases and controls in subjects with metabolic syndrome was significant. No significant difference in QoL parameters was observed between cases and controls in subjects without metabolic syndrome.
Richard $\mathrm{R}$ et al in his review reported that quality of life was worse for people with diabetes compared to the general population, especially regarding physical functioning and well-being. ${ }^{3}$ No difference was observed in QOL domain scores for type-2 patients and the general population group in two domains (social and environmental) indicates that disease has an impact on QOL and it varies with disease severity. Whereas no significant impact was observed for of duration of illness. $^{12-14}$

\section{CONCLUSION}

Quality of life with respect to physical, psychological and social domain among diabetics with metabolic syndrome was reduced significantly. Hence metabolic disease plays an important role in quality of life among diabetic subjects.

\section{ACKNOWLEDGEMENTS}

Authors would like Dr. Krishnamurthy (PRO), Interns and Postgraduates in Department of Community Medicine for there contributions in collection of data.

Funding: No funding sources

Conflict of interest: None declared

Ethical approval: The study was approved by the Institutional Ethics Committee

\section{REFERENCES}

1. Nagpal J, Kumar A, Kakar S, Bhartia A. The development of quality of life instrument for Indian diabetes patients (QOLID): a validation and reliability study in middle and higher income groups. J Asso Physicians India. 2010;58:295-304.

2. Fal AM, Jankowska B, Uchmanowicz I. Type 2 diabetes quality of life patients treated with insulin and oral hypoglycemic medication. Bio Med Central. 2011;48(3):237-42.

3. Richard R, Rubin CDE. Diabetes and quality of life. Diabetes Spectrum. 2000;13:21.

4. Orley J. Introduction, administration, scoring and generic version of the assessment field trial version. Programme on mental health. World Health Organization. 1996.

5. The World Health Organization Quality of Life (WHOQOL)-BREF. World Health Organization 2004.

6. World Health Organization. Definition, diagnosis and classification of diabetes mellitus and its complications: Report of a WHO Consultation. Available at: http:// www.whqlibdoc.who.int/hq/1999/WHO_NCD_NC S_99.2.pdf. Accessed on 11January 2016.

7. The IDF consensus worldwide definition of the metabolic syndrome 2006. Available from: http://www.idf.org/webdata/docs/IDF_Meta_def_fin al.pdf . Accessed on 15 June 2016. 
8. Misra A, Wasir JS, Pandey RM. An evaluation of candidate definitions of the metabolic syndrome in adult Asian Indians. Diabetes Care. 2005;28:398-03.

9. Srinivas HK, Venkatesha M, Prasad R. Quality of life assessment among type 2 diabetic patients in rural tertiary centre. Int J Med Sci Public Health. 2014;3(3);405-7.

10. Javanbakht M, Abolhasani F, Mashayekhi A, Baradaran HR, Jahangiri Y. Health related quality of life in patients with type 2 diabetes mellitus in Iran: A national survey. PLOS ONE. 2012;7(8):44526.

11. Rubin RR, Peyrot M. Quality of life and diabetes. Diabetes Metab Res Rev. 1999;15:205-18.
12. Burroughs TE, Desikan R, Waterman BM. Development and validation of the diabetes quality of life brief clinical inventory. Diabetes Spectrum. 2004;17:41-9.

13. UK prospective diabetes study group. quality of life in type 2 diabetes patients is affected by complications but not by intensive policies to improve blood glucose or blood pressure control (UKPDS 37). Diabetes Care. 1999;22:1125-36.

Cite this article as: Mahesh V, Latha $\mathrm{K}$, Muninarayana C, Ravishankar S. Quality of life in diabetic subjects with respect to metabolic syndrome: a case control study. Int J Community Med Public Health 2016;3:2393-6. 